\title{
Efecto de la densidad de plantación sobre crecimiento, producción y calidad en cinco accesiones de aji (Capsicum spp.) cultivadas en la Amazonia occidental colombiana
}

\section{Effect of planting density on growth, production and quality of five accessions of hot pepper (Capsicum spp.) in the Colombian Western Amazon}

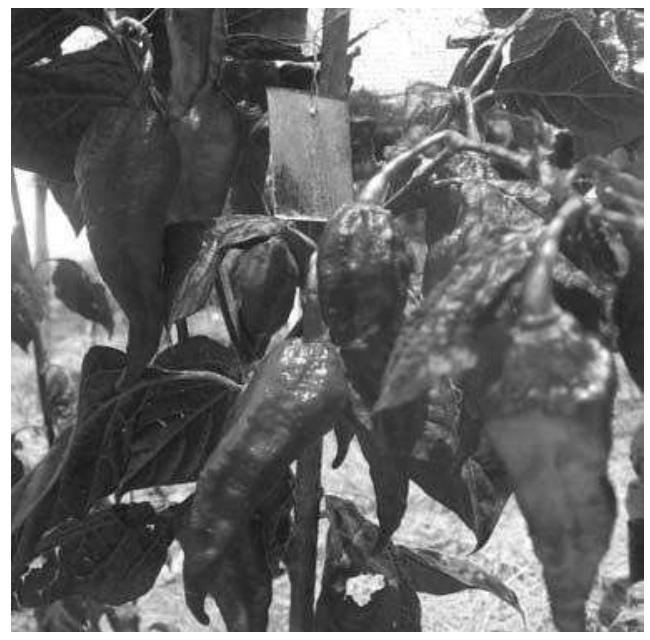

CAMILA CASTELLANOS ${ }^{1}$

JAIME A. BARRERA ${ }^{2}$

MARÍA SOLEDAD HERNÁNDEZ2,6

LUZ MARINA MELGAREJO ${ }^{3}$

MARCELA CARRILLO²

LIGIA RODRÍGUEZ ${ }^{4}$

ORLANDO MARTÍNEZ ${ }^{5}$

Ají amazónico en fructificación.

Foto: J.A. Barrera

\section{RESUMEN}

El género Capsicum, perteneciente a la familia Solanaceae, presenta condiciones favorables para un buen desarrollo en la Amazonia colombiana. De la colección de germoplasma de Capsicum Amazónico del Instituto Sinchi se seleccionaron cinco accesiones por su alto grado de pungencia con el objeto de evaluar su crecimiento y el efecto sobre los patrones de interceptación de la radiación fotosintéticamente activa (RFA), producción y calidad de los frutos bajo tres distancias de siembra. Para este propósito se estableció un experimento en condiciones de campo en la ciudad de Florencia, Caquetá. Durante las nueve semanas del estudio, los índices de crecimiento en general mostraron una producción de biomasa de manera eficiente y sostenida en todas las accesiones. No se presentaron diferencias significativas en producción y en la radiación interceptada bajo diferentes distancias de siembra, pero sí entre las accesiones, siendo la CS 285 la que logra mayor porcentaje de interceptación de RFA, asociado este resultado a la arquitectura que exhiben las accesiones. Para las cinco accesiones la TAN exhibió su máximo valor entre los 27 y 41 días después de transplante.

Departamento de Agronomía, Facultad de Agronomia, Universidad Nacional de Colombia, Bogotá (Colombia).

Instituto Amazónico de Investigaciones Científicas (Sinchi), Bogotá (Colombia).

Departamento de Biología, Facultad de Ciencias, Universidad Nacional de Colombia, Bogotá (Colombia).

Facultad de Ciencias Naturales, Universidad de Bogotá Jorge Tadeo Lozano, Bogotá (Colombia).

Facultad de Ciencias, Universidad de los Andes, Bogotá (Colombia).

Autor para correspondencia.shernandez@sinchi.org.co 


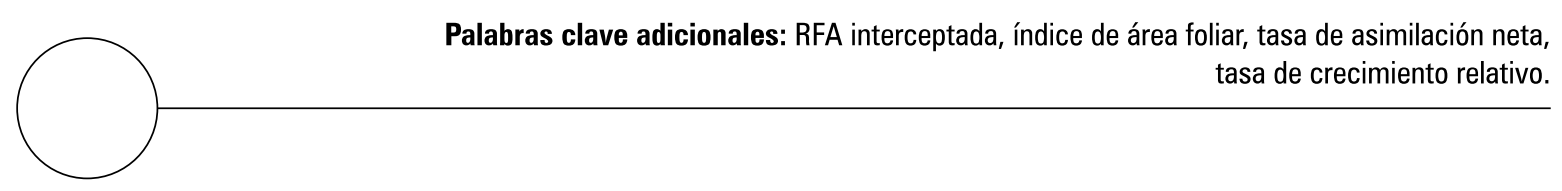

\section{ABSTRACT}

The genus Capsicum belonging to Solanaceae family enjoys favorable conditions for successful development in the Colombian Amazon. Five accessions of the Germplasm collection of Amazonian Capsicum of the Institute SINCHI were selected for their high pungency to study the growth, development and the effect on the patterns of interception of photosynthetic active radiation (PAR), production and quality of fruits under three planting distances $(50,60$ and $70 \mathrm{~cm}$ between plants). For this purpose, an experiment was conducted in field conditions in Florencia, Caqueta. Growth rates, in general, showed an efficient and sustained production of biomass in all accessions in response to the strengthening of their photosynthetic apparatus during the growth. There were no significant differences among the plants in production and intercepted radiation under different distances, but among the accessions it was the CS 285 that achieves the highest percentage PAR interception; this result is associated with the architecture that have the accessions. The five accessions showed highest NAR rate between 27 and 41 days after transplanting.

Additional keywords: intercepted PAR, leaf area index, net assimilation rate, relative growth rate.

Bolivia, Perú, el Sur de México y Colombia son países considerados como el lugar de origen del género Capsicum -uno de los más importantes de la familia de las Solanaceae-, que comprende cerca de 25 especies silvestres y cinco domesticadas, incluyendo todas la variedades de ají y pimentón. Las cinco especies domesticadas $y$ cultivadas comercialmente son $C$. annuum, $C$. frutescens, C. baccatum, C. pubescens y C. chinense (IBPGR, 1983).

El Instituto Amazónico de Investigaciones Científicas, Sinchi, tiene a su cuidado un banco de germoplasma de Capsicum ex situ con una colección de 472 materiales de la región amazónica (Méndez et al., 2004) con diferente contenido de pungencia, característica propia de los ajíes. Según Villachica (1996), la Amazonia presenta condiciones favorables para la producción continua de ají durante el año, lo que podría servir para abastecer el mercado de los países con invierno prolongado.

Los factores que influyen la respuesta fisiológica frente a la densidad de plantas, viendo ésta como un factor cambiante, se pueden comparar mediante la influencia de la radiación solar durante la competencia de las plantas por recursos disponibles (Rodríguez et al., 2004). La respuesta de las plantas frente a la densidad de siembra o por competencia con otras es comparativa frente al efecto de la radiación solar. Para comprender cómo afecta la luz a la tasa fotosintética, hay que considerar que las longitudes de onda comprendidas entre 400 y $700 \mathrm{~nm}$ son la porción del espectro que las plantas usan para la fotosínte- 
sis, las cuales se conocen como radiación fotosintéticamente activa (RFA). Esta radiación ha sido establecida como uno de los factores determinantes para calcular los rendimientos potenciales de los cultivos, ya sean densos o sembrados en arreglos especiales (Castillo et al., 1997). La disponibilidad de la radiación solar necesaria para los procesos biológicos que ocurren en un cultivo está determinada en primer término por el macroclima de la región (latitud y día del año), por las condiciones de nubosidad que determinan las cantidades de radiación directa y difusa y por las propiedades del follaje, como el tamaño y la disposición de las hojas, estructura de la planta y distancia de siembra (Jaramillo-Robledo, 2005). Una correcta y apropiada distribución de radiación solar, entre y dentro de los doseles, dará como resultado un trabajo más homogéneo del índice de área foliar (IAF), mejor aprovechamiento de la luz, aumento en la eficiencia fotosintética, menos respiración de mantenimiento $\mathrm{y}$, por tanto, mayores rendimientos agronómicos (Lee et al., 2000). El incremento en la densidad de siembra es uno de los principales manejos que se hacen para mejorar la captura de radiación solar por los cultivos (Idinoba et al., 2002).

El rendimiento y la producción de las plantas cultivadas puede medirse mediante el empleo de índices de crecimiento, los cuales indican la eficacia de las plantas para aprovechar los factores ambientales del sitio donde crecen y la forma en que las plantas distribuyen sus asimilados (Méndez et al., 2004). Las diferentes estrategias de manejo durante el ciclo de vida de un cultivo pueden producir efectos en el crecimiento y desarrollo de las plantas; en algunos casos, como el del pimentón (C. annuum L.), hay variaciones que pueden ser inherentes a los diversos genotipos o pueden ser modificadas por factores, como la distancia de siembra entre las plantas (Viloria et al., 1998). En evaluaciones anteriores de material del Instituto Sinchi, Méndez et al. (2003) observaron que el aborto de frutos se encuentra influenciado por la alta densidad de siembra, generando una alta competencia por los nutrientes y luz entre plantas y por fotoasimilados por parte de los frutos. También se ha encontrado que las distancias de siembradesempeñan un papel determinante en la intercepción de radiación solar por el cultivo para convertir esta energía en biomasa, sin dejar de lado otros factores importantes, como el cultivar elegido y sus características de crecimiento, al igual que las características climáticas del lugar donde se va a desarrollar el cultivo (Carrillo et al., 2003).

En esta investigación se determinó el efecto que la densidad de siembra ejerce sobre el crecimiento y desarrollo de cinco accesiones de ají amazónico (Capsicum sp.) colección Sinchi cultivadas bajo condiciones de la Amazonia occidental y compara su capacidad individual de interceptación de radiación (RFA).

\section{MATERIALES Y MÉTODOS}

El trabajo se realizó en la granja experimental Santo Domingo de la Universidad de la Amazonia a $7 \mathrm{~km}$ de Florencia vía Morelia (Departamento de Caquetá, Colombia), caracterizada con una temperatura promedio anual de $26^{\circ} \mathrm{C}$, humedad relativa entre 70 y $80 \%$ y precipitación de 2.500 a $3.300 \mathrm{~mm}$ año-1.

En el ensayo se empleó un diseño de parcelas divididas distribuidas completamente al azar con estructura factorial de tres densidades de siembra y cinco accesiones en tres repeticiones, para un total de 15 tratamientos, que se describen en la tabla 1. Las accesiones fueron seleccionadas por su alto nivel de pungencia (tabla 2). Las plántulas de $15 \mathrm{~cm}$ de altura se sembraron en suelo, en camas de $0,9 \mathrm{~m}$ de ancho por $18 \mathrm{~m}$ de largo con una polisombra de $30 \%$ a $1,5 \mathrm{~m}$ de altura del suelo. Para el mantenimiento nutricional en cada parcela se empleó abonamiento orgánico con Bocachi, en aplicación al suelo alrededor de la planta en dosis de $500 \mathrm{~g}$ por planta cada dos meses. 
Tabla 1. Tratamientos realizados en el ensayo experimental.

\begin{tabular}{|c|c|c|}
\hline 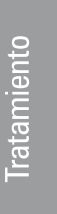 & 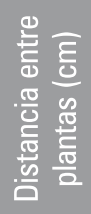 & 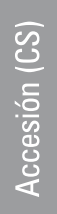 \\
\hline T1 & 50 & 009 \\
\hline $\mathrm{T} 2$ & 50 & 210 \\
\hline T3 & 50 & 219 \\
\hline T4 & 50 & 285 \\
\hline T5 & 50 & 469 \\
\hline
\end{tabular}

\begin{tabular}{|c|c|c|}
\hline 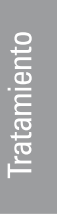 & 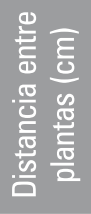 & $\begin{array}{l}\overline{\mathbb{S}} \\
\text { :을 } \\
\text { dु } \\
\frac{0}{1}\end{array}$ \\
\hline T6 & 60 & 009 \\
\hline T7 & 60 & 210 \\
\hline T8 & 60 & 219 \\
\hline T9 & 60 & 285 \\
\hline T10 & 60 & 469 \\
\hline
\end{tabular}

\begin{tabular}{|c|c|c|}
\hline 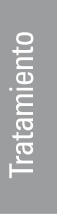 & 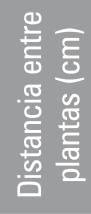 & 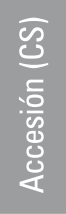 \\
\hline T11 & 70 & 009 \\
\hline T12 & 70 & 210 \\
\hline $\mathrm{T} 13$ & 70 & 219 \\
\hline $\mathrm{T} 14$ & 70 & 285 \\
\hline T15 & 70 & 469 \\
\hline
\end{tabular}

CS, Colección Sinchi.

\begin{tabular}{|c|c|c|}
\hline Accesión & Especie & Pungencia USH \\
\hline CS 210 & C. chinense & 53.068 \\
\hline CS 469 & C. chinense & 19.000 \\
\hline CS 219 & C. annuum & 17.500 \\
\hline CS 285 & C. frutescens & 16.000 \\
\hline CS 009 & C. chinense & 12.100 \\
\hline
\end{tabular}

USH, Units Scoville Heats (Unidades de pungencia).

Dos semanas después de sembradas las plantas se iniciaron evaluaciones semanales, durante un lapso de nueve semanas, tiempo en el cual se alcanzó el ciclo de cosecha. Se registraron las variables de altura de la planta y área del dosel, diámetro basal del tallo con el uso de un calibrador, peso seco de la planta completa mediante secado en horno de circulación de aire a $75^{\circ} \mathrm{C}$ por un periodo de $72 \mathrm{~h}$, área foliar utilizando un planímetro digital marca LI-COR ${ }^{\circledR}$. La radiación fotosintéticamente activa (RFA) se midió en la segunda floración y fructificación de las plantas con un Ceptómetro AccuPAR ${ }^{\circledR}$ Modelo LP-80. Estos datos se tomaron sobre y bajo el dosel de las plantas, ubicando la barra de tal forma que abarcara dos de las cuatro plantas de cada unidad experimental. Los índices de crecimiento se calcularon utilizando las fórmulas propuesta por Gardner et al. (1985) (tabla 3). En la cosecha, 48 d después de trasplante, se colectaron los frutos para determinar el número de frutos y su peso fresco.

Tabla 3. Fórmulas para calcular los índices de crecimiento (Gardner et al., 1990).

\begin{tabular}{|c|c|c|}
\hline Índice de crecimiento & Ecuación & Unidades \\
\hline Índice de área foliar (IAF) & $\left(\frac{A F_{2}+A F_{1}}{2}\right) \cdot\left(\frac{1}{\mathrm{AS}}\right)$ & sin unidad \\
\hline Tasa de asimilación neta (TAN) & $\left(\frac{\mathrm{P}_{2}-\mathrm{P}_{1}}{\mathrm{t}_{2}-\mathrm{t}_{1}}\right) \cdot\left(\frac{\left(L n A F_{2}-L n A F_{1}\right)}{A F_{2}-A F_{1}}\right)$ & $\mathrm{g} \mathrm{cm}^{-2} \mathrm{día}^{-1}$ \\
\hline Duración de área foliar (DAF) & $\frac{\left(I A F_{2}-I A F_{1}\right) \bullet\left(\mathrm{t}_{2}-\mathrm{t}_{1}\right)}{2}$ & días \\
\hline Tasa de crecimiento relativo (TCR) & $\frac{\left(\operatorname{Ln} P_{2}-\operatorname{Ln} P_{1}\right)}{\mathrm{t}_{2}-\mathrm{t}_{1}}$ & $\mathrm{~g} \mathrm{~g}^{-1} \mathrm{di}^{-1}{ }^{-1}$ \\
\hline Tasa de crecimiento del cultivo (TCC) & $\frac{1}{\mathrm{AS}} \cdot\left(\frac{\mathrm{P}_{2}-\mathrm{P}_{1}}{\mathrm{t}_{2}-\mathrm{t}_{1}}\right)$ & $g^{-2}$ día $^{-1}$ \\
\hline \multicolumn{3}{|c|}{$\begin{array}{c}A F_{2}=\text { Área foliar final; } A F_{1}=\text { Área foliar inicial; } A S=\text { Área del dosel; } P_{2}=\text { Peso final; } P_{1}=\text { Peso inicial; } t_{2}=\text { Tiempo final; } \\
\qquad t_{1}=\text { Tiempo inicial; } I A F_{2}=\text { Índice de área foliar final; } I A F_{1}=\text { Índice de área foliar inicial. }\end{array}$} \\
\hline
\end{tabular}


La calidad de los frutos se midió de acuerdo con el contenido de capsaicinoides y con base en la metodología reportada por Barrera et al. (2008), tomando una muestra de $6,25 \mathrm{~g}$ de frutos dispuestos en $50 \mathrm{~mL}$ de alcohol al $96 \%$, sometidos a extracción durante $5 \mathrm{~h}$, al cabo de las cuales se filtra el líquido y se pone en un Rotavapor Heidolph ${ }^{\circledR}$ LABOROTA 4000-Efficient a $90 \mathrm{rpm}$ y $75^{\circ} \mathrm{C}$ hasta obtener $10 \mathrm{~mL}$ de extracto. Posteriormente se envasó en frasco ámbar rotulado y se realizó la cuantificación del contenido de capsaicinoides por el sistema CLAE (HPLC) con columna Zorbax Eclipse XDB-C18 a $40^{\circ} \mathrm{C}$. La fase móvil utilizada fue una mezcla de acetonitrilo, ácido acético y agua en la proporción 40:59:1 (v: v) con un flujo de $1,0 \mathrm{~mL} \mathrm{~min}^{-1}$. Se utilizó como patrón 8-metil-n-vanilil-6-nonenamida (Sigma, St. Louis, MO). Los datos se expresaron en USH (Unidades Scoville de calor), usando el valor de Todd reportado por Sinan et al. (2005).

La información obtenida se sometió a análisis de varianza y las diferencias entre los tratamientos se establecieron mediante la prueba de diferencia significativa mínima (LSD) con un alfa de 0,05. Los valores de área foliar y peso seco se procesaron mediante análisis de regresión exponencial. Se utilizó el paquete estadístico Statitistix 8.0.

\section{RESULTADOS Y DISCUSIÓN}

\section{Análisis de crecimiento}

En la figura 1 se observa el rápido aumento del área foliar entre los días 41 y 69 después de trasplante ddt, coincidiendo con el comienzo de la etapa de fructificación, en la que la planta debe obtener fotoasimilados para empezar el llenado del fruto. Los promedios más altos se dieron para las accesiones CS 219 (C. annuum) y CS 210 (C. chinense). Probablemente, los factores como la RFA movieron asimilación neta de la planta, de esta manera la planta asignó materia seca hacia las hojas entre los $40 \mathrm{ddt}$ y $70 \mathrm{ddt}$.

El peso seco (figura 2) aumenta rápidamente durante estos intervalos de tiempo, debido a la presencia de los nuevos frutos. Los modelos que explican el comportamiento del área foliar y el peso seco de las accesiones se aprecian en la tabla 4. Los resultados (figura 1 y 2 ) muestran que la fase de crecimiento rápido se presenta después de los $41 \mathrm{ddt}$ para las accesiones CS 009, CS 210 y CS 469 (C. chinense), de los $27 \mathrm{ddt}$ para la accesión CS 285 (C. frutescens) y de los 34 ddt para la accesión CS 219 (C. annuum).

Tabla 4. Ecuaciones de regresión para los valores de área foliar y peso seco de las cinco accesiones de ají Capsicum spp.

\begin{tabular}{|c|c|c|c|}
\hline \multirow{2}{*}{ Accesión } & Variable & Ecuación & $r^{2}$ \\
\hline \multirow{2}{*}{ CS 009 } & Área foliar & $y=0,3438 \times 2-13,431 \times+191,46$ & 0,9148 \\
\hline \multirow{2}{*}{ CS210 } & Peso seco & $y=0,00625 \times 2-0,2546 \times+3,4455$ & 0,8762 \\
\hline \multirow{2}{*}{ CS 219 } & Área foliar & $y=0,2536 \times 2-5,4358 x+63,446$ & 0,8214 \\
\cline { 2 - 4 } & Peso seco & $y=0,00565 \times 2-0,1246 x+1,6179$ & 0,8484 \\
\hline \multirow{2}{*}{ CS 285 } & Área foliar & $y=0,4004 \times 2+12,985 x+145,18$ & 0,7969 \\
\hline \multirow{2}{*}{ CS 469 } & Peso seco & $y=27,505 \times 2+4,1600 x+1,7404$ & 0,8718 \\
\cline { 2 - 4 } & Área foliar & $y=0,2016 \times 2-1,2705 x-1,732$ & 0,8399 \\
\cline { 2 - 4 } & Peso seco & $y=0,0032 \times 2-0,0602 x+1,7064$ & 0,8240 \\
\hline
\end{tabular}



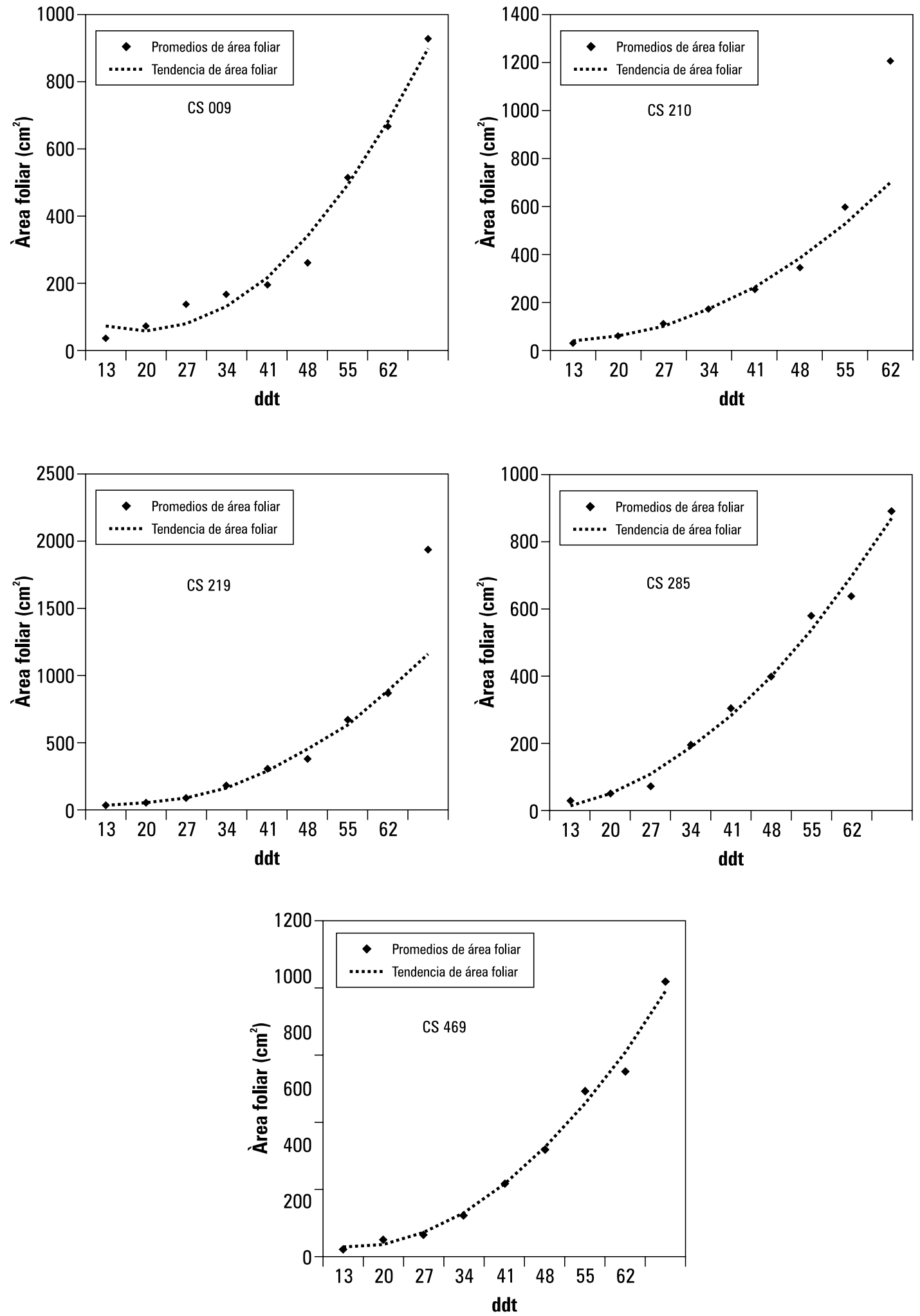

Figura 1. Área foliar en cinco accesiones de ají Capsicum sp. de la colección Sinchi establecidas en Florencia, Caquetá. 

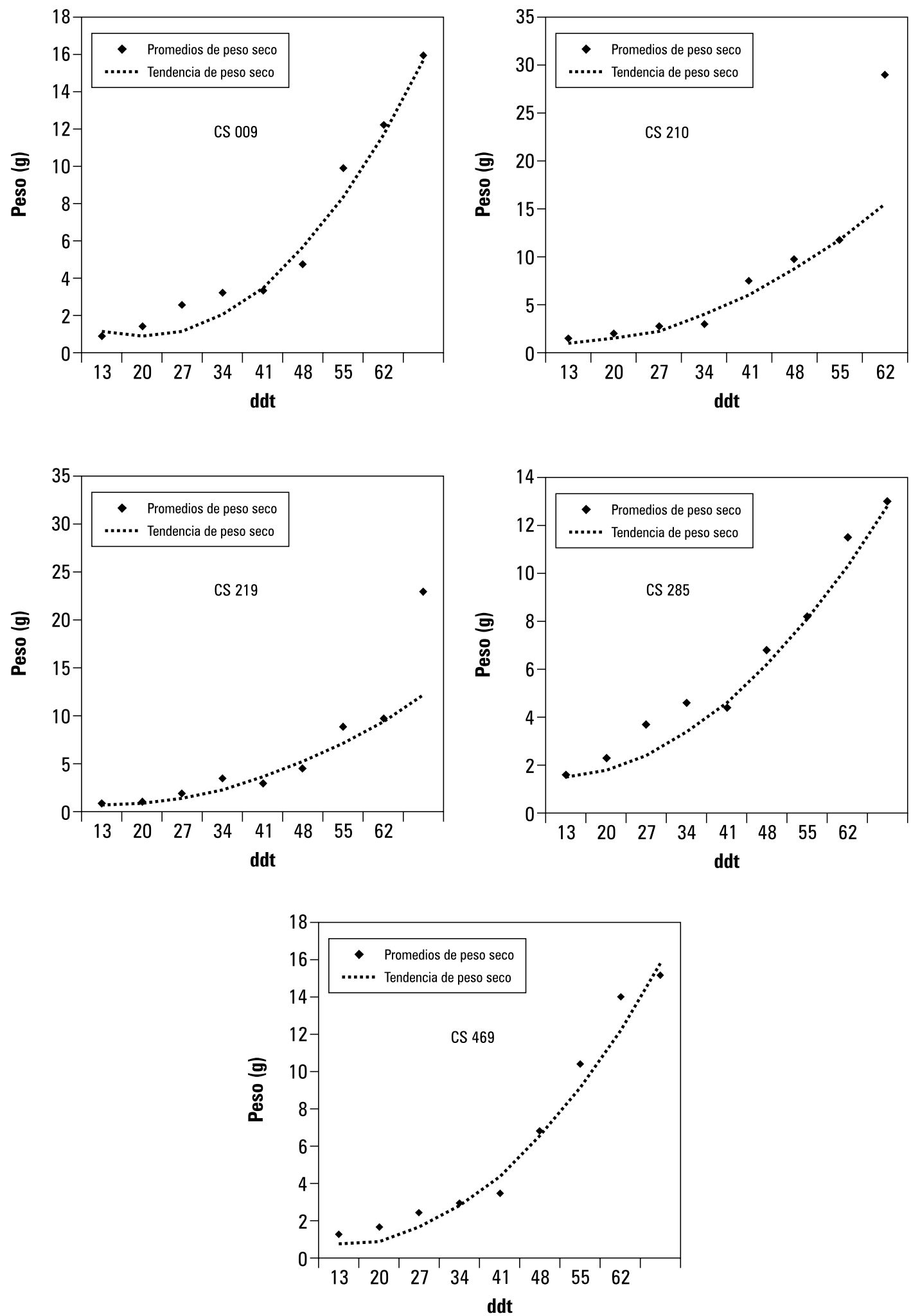

Figura 2. Peso seco en cinco accesiones de ají Capsicum spp. de la colección Sinchi establecidas en Florencia, Caquetá. 


\section{Indices de crecimiento}

Los índices de crecimiento de cinco accesiones de ají amazónico presentaron una tendencia ascendente entre los 13 ddt y los 69 ddt (tabla 5) que está acorde con el comportamiento de los índices de crecimiento de un cultivo anual que crece bajo libre exposicion En condiciones de cultivo, de manera particular a partir de los $27 \mathrm{ddt}$, el comportamiento de la TCR sigue una tendencia acorde con lo observado para muchas especies de crecimiento determinado, teniendo los valores altos que disminuyen con la edad; esta disminución se debe al aumento gradual de tejidos no asimilatorios (Jarma et al., 1999).

La tasa de acumulación de materia seca (MS) por unidad de área foliar y tiempo se define como la TAN, que es una medida de la eficiencia media fotosintética de las hojas (Mansab et al., 2003). Para las cinco accesiones, la TAN exhibe su máximo valor entre los $27 \mathrm{ddt}$ y los $41 \mathrm{ddt}$. Hunt (2003) indica que la TAN exhibe en general un nivel constante relativo, modificado por fluctuaciones en el ambiente durante el periodo de crecimiento. Páez et al. (2000) encontraron en una Solanacea como el tomate var. Río Grande una TAN mayor en los primeros estados de desarrollo de plantas expuestas a radiación total, condición que se hizo contraria en los estados más avanzados de crecimiento.

Los resultados anteriores sugieren que, en términos generales, en las etapas vegetativas de las especies anuales la planta produce biomasa de manera eficiente y sostenida en respuesta a la capacidad fotosintética. Esto explicaría la tendencia similar que muestran los índices de crecimiento IAF, TAN y TCR de los materiales de ají en etapas tempranas de crecimiento. Jarma et al. (2006) reportan que esta eficiencia, ante la diversidad de estímulos ambientales y genéticos, se vuelve diferencial en las fases tardías, cuando la planta ha alcanzado su madurez fisiológica.

\begin{tabular}{|c|c|c|c|c|c|}
\hline Accesión & ddt & IAF & TAN & DAF & TCR \\
\hline \multirow{5}{*}{ CS 009} & 13 & 0,5041 & - & - & - \\
\hline & 27 & 0,9895 & 0,0019 & 6,8825 & $-0,0004$ \\
\hline & 41 & 0,6967 & 0,0000 & 5,1844 & 0,0533 \\
\hline & 55 & 0,9520 & 0,0020 & 5,8311 & 0,1015 \\
\hline & 69 & 0,4816 & 0,0006 & 3,6290 & 0,0380 \\
\hline \multirow{5}{*}{ CS 210} & 13 & 0,3038 & - & 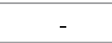 & 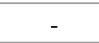 \\
\hline & 27 & 0,6682 & 0,00162 & 4,7254 & 0,0647 \\
\hline & 41 & 1,7452 & 0,00329 & 9,9624 & 0,1365 \\
\hline & 55 & 1,9887 & 0,00051 & 17,0251 & 0,0212 \\
\hline & 62 & 6,6095 & 0,00482 & 43,1604 & 0,1957 \\
\hline \multirow{5}{*}{ CS 219} & 13 & 0,7172 & - & - & - \\
\hline & 27 & 0,9244 & 0,0062 & 6,033 & 0,0878 \\
\hline & 41 & 1,0669 & $-0,0009$ & 7,681 & $-0,0252$ \\
\hline & 55 & 2,0962 & 0,0019 & 13,473 & 0,0948 \\
\hline & 69 & 5,2270 & 0,0032 & 35,502 & 0,1729 \\
\hline \multirow{5}{*}{ CS 285} & 13 & 0,2886 & - & - & - \\
\hline & 27 & 0,6782 & 0,0047 & 3,875 & 0,0614 \\
\hline & 41 & 1,0638 & $-0,0002$ & 7,435 & $-0,0023$ \\
\hline & 55 & 0,9377 & 0,0004 & 7,482 & 0,0277 \\
\hline & 69 & 0,5500 & 0,0004 & 6,041 & 0,0209 \\
\hline \multirow{5}{*}{ CS 469} & 13 & 0,3062 & - & - & - \\
\hline & 27 & 0,4469 & 0,0021 & 2,903 & \\
\hline & 41 & 0,8418 & 0,0003 & 5,316 & \\
\hline & 55 & 0,9898 & 0,0010 & 7,683 & \\
\hline & 69 & 0,4554 & 0,0002 & 5,114 & \\
\hline
\end{tabular}


Efecto de la densidad de siembra sobre la RAF, producción y calidad de frutos

En las densidades de siembra evaluadas (figura 3) se observa la evolución de la altura durante las nueve semanas de ciclo de cultivo para las cinco accesiones. Contrario a lo reportado, a mayor densidad de siembra, esto es, a distancia de 50 $\mathrm{cm}$ entre plantas, se observaron los valores más bajos de altura para las accesiones CS 009, CS 210, CS 219 y CS 295. Experiencias previas indican que la posibilidad de usar pequeñas distancias de siembra en plantas del género Capsicum es un poco limitada, ya que las altas densidades traen como respuesta un incremento en la altura (Decoteau y Hatt Graham, 1994), como lo observado para la accesión CS 469 (C. chinense). Generalmente, los incrementos en la altura de la planta, en respuesta al aumento en la densidad de población, son indicativos de una mayor competencia por luz entre plantas, debido a menor disponibilidad de radiación dentro del dosel (Castillo et al., 1997).

Las diferencias significativas se encontraron a la densidad de siembra más baja en las accesiones CS 009 y CS 285 donde se registraron los mayores valores de altura y no hubo diferencias en las densidades medias y altas $(50 \mathrm{~cm}$ y $60 \mathrm{~cm}$ entre plantas). En el caso de la accesión CS 210, se presentan diferencias significativas en la densidad media, siendo ésta en la que se alcanzó la mayor altura. La accesión CS 219 presentó diferencias significativas entre las tres distancias, obteniendo la mayor altura a la menor densidad de siembra. La accesión CS 469 presenta diferencias significativas para la menor densidad de siembra, en la que se alcanzó el menor valor de altura (figura 3). La interacción genotipo y ambiente determinó la respuesta obtenida en cuatro de las cinco accesiones porque las plantas de las especies chinense, frutescens y annuum difieren en cuando a la arquitectura. De acuerdo con Nuez y Costa (1996) y los estudios morfo- lógicos adelantados por Melgarejo et al. (2004), las plantas de ají varían ampliamente en forma y tamaño, encontrando desde herbáceas hasta arbustos entre $25 \mathrm{~cm}$ y $190 \mathrm{~cm}$ de altura; son plantas semiarbustivas perennes, pero de cultivo anual, y alcanzan entre los 0,3 y 1,5 m de altura, dependiendo principalmente de la variedad, de las condiciones climáticas y de la fertilización.

El diámetro basal del tallo fue mayor en las plantas sembradas a menor densidad con excepción de la accesión CS 469 (C. chinense). Díaz et al. (1999) obtuvieron una disminución de diámetro del tallo a mayor altura en las plantas de pimentón. Sin embargo, el comportamiento del diámetro en la accesión CS 469 no muestra diferencias significativas entre ninguna de las distancias de siembra. La accesión CS 219 (C. annuum) presentó diferencias significativas entre todas las distancias, mientras que en las accesiones CS 009 y CS 285 existieron diferencias significativas a la distancia $70 \mathrm{~cm}$. Finalmente, en la accesión CS 210, aunque el mayor valor es alcanzado en la distancia de $70 \mathrm{~cm}$, la diferencia significativa sólo se presenta entre esta distancia y la de $50 \mathrm{~cm}$ (figura 4). En general, una mayor exposición a radiación solar favorecerá el crecimiento de la planta, lo cual se observó en casi todas las accesiones.

\section{Producción y calidad de frutos}

Se presentaron diferencias significativas para la variable número de frutos (figura 5) entre la accesión CS 285 (C. frutescens) y las demás accesiones. La accesión CS 219 (C. annuum) sólo presentó diferencias significativas con la accesión CS 285. Entre las accesiones de C. chinense, CS 009 y CS210 no se presentaron diferencias significativas, pero entre éstas y la accesión CS 469 (C. chinense) se las presentaron. Mendez et al. (2003) evaluando accesiones de ají amazónico en condiciones de Leticia, Amazonas, encontraron respuestas diferentes en producción de frutos entre materiales de C. chinense y C. baccatum. 

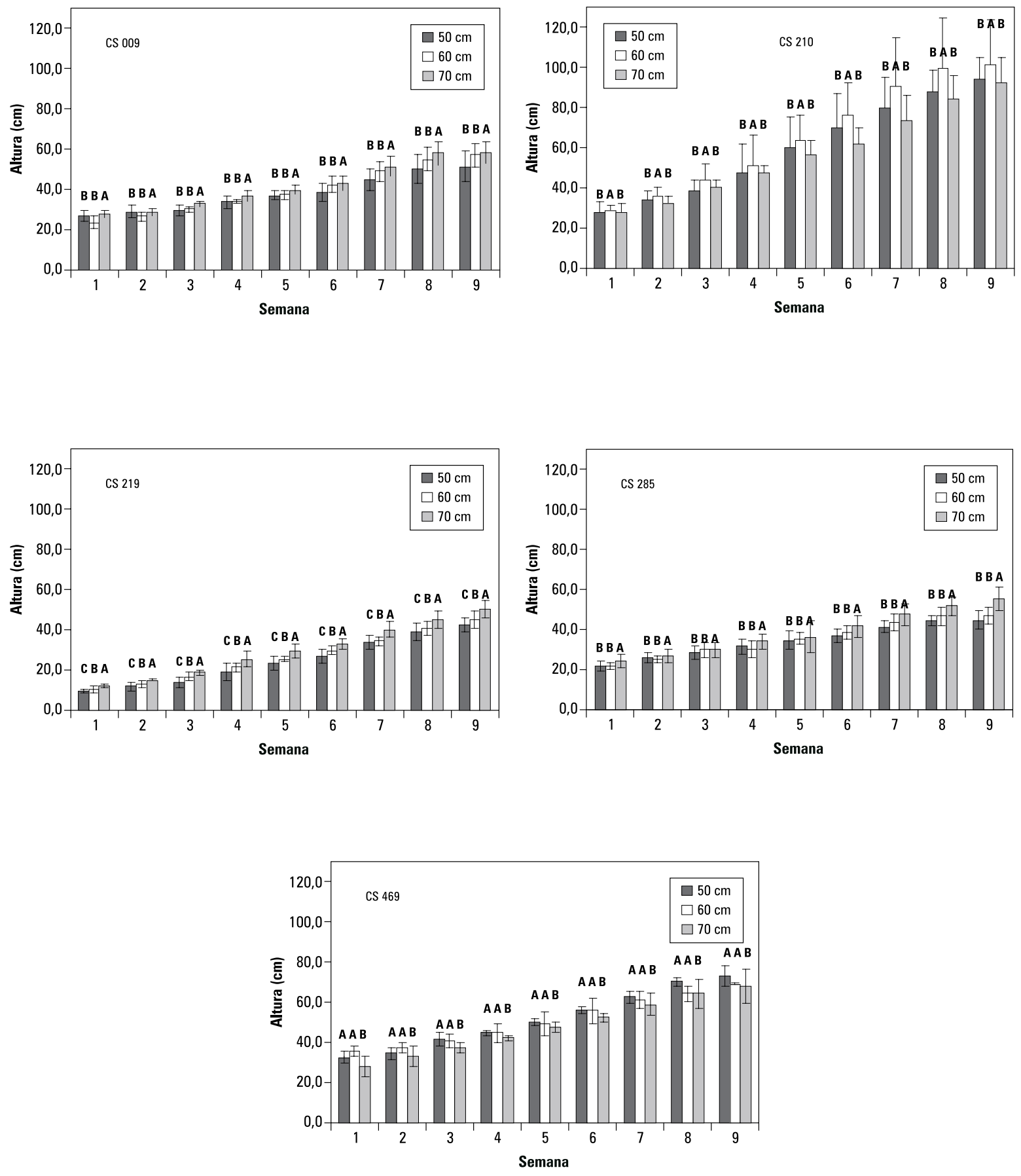

Figura 3. Comportamiento de la altura de la planta en cinco accesiones de ají Capsicum spp. con tres distancias de siembra. Promedios con letras distintas en cada semana indican diferencia significativa según la prueba de LSD $(P \leq 0,05)$. Las barras sobre las columnas indican desviación estándar. 

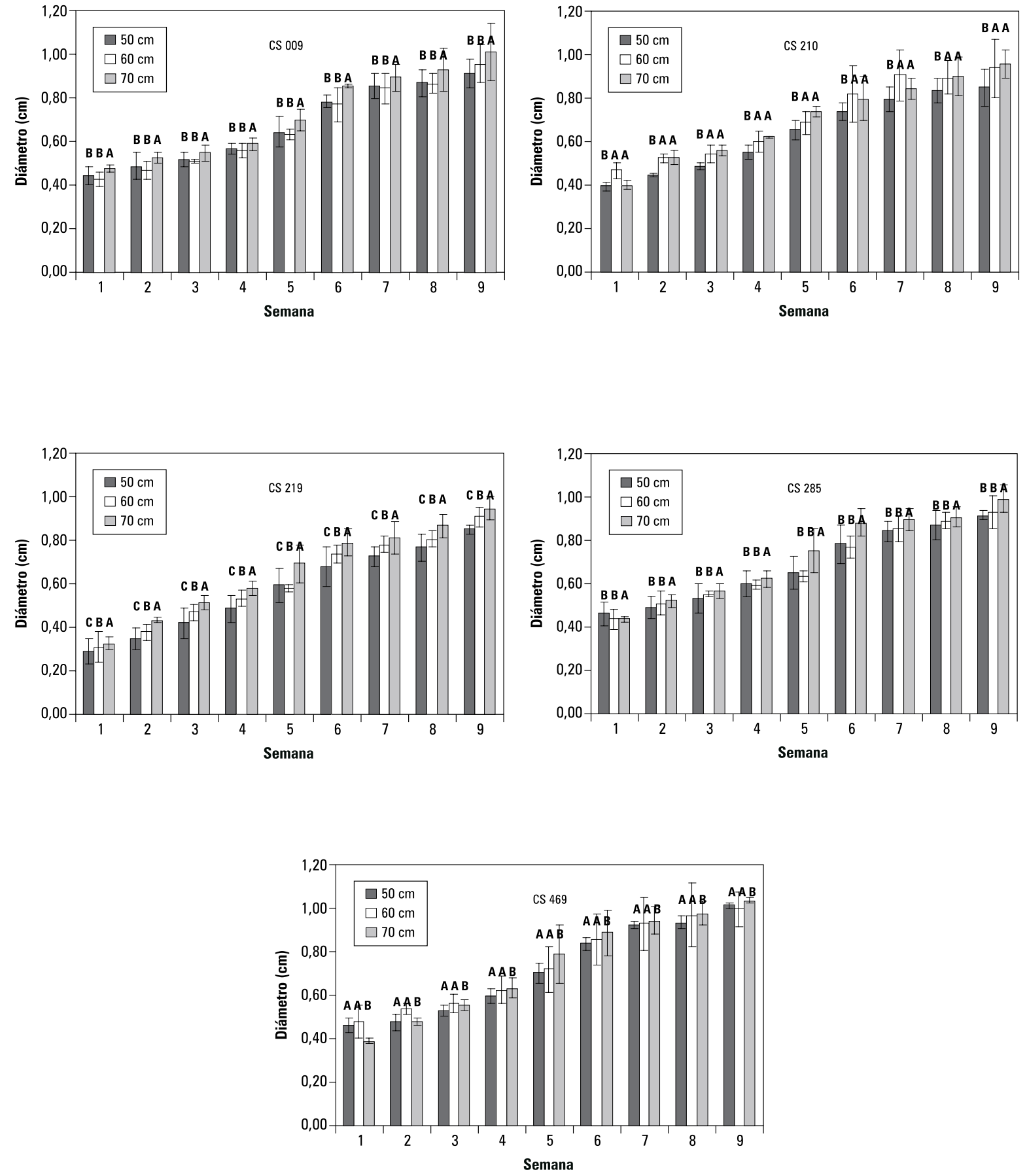

Figura 4. Comportamiento del diámetro basal del tallo en cinco accesiones de aji Capsicum spp. con tres distancias de siembra. Promedios con letras distintas en cada semana indican diferencia significativa según la prueba de LSD $(P \leq 0,05)$. Las barras sobre las columnas indican desviación estándar. 


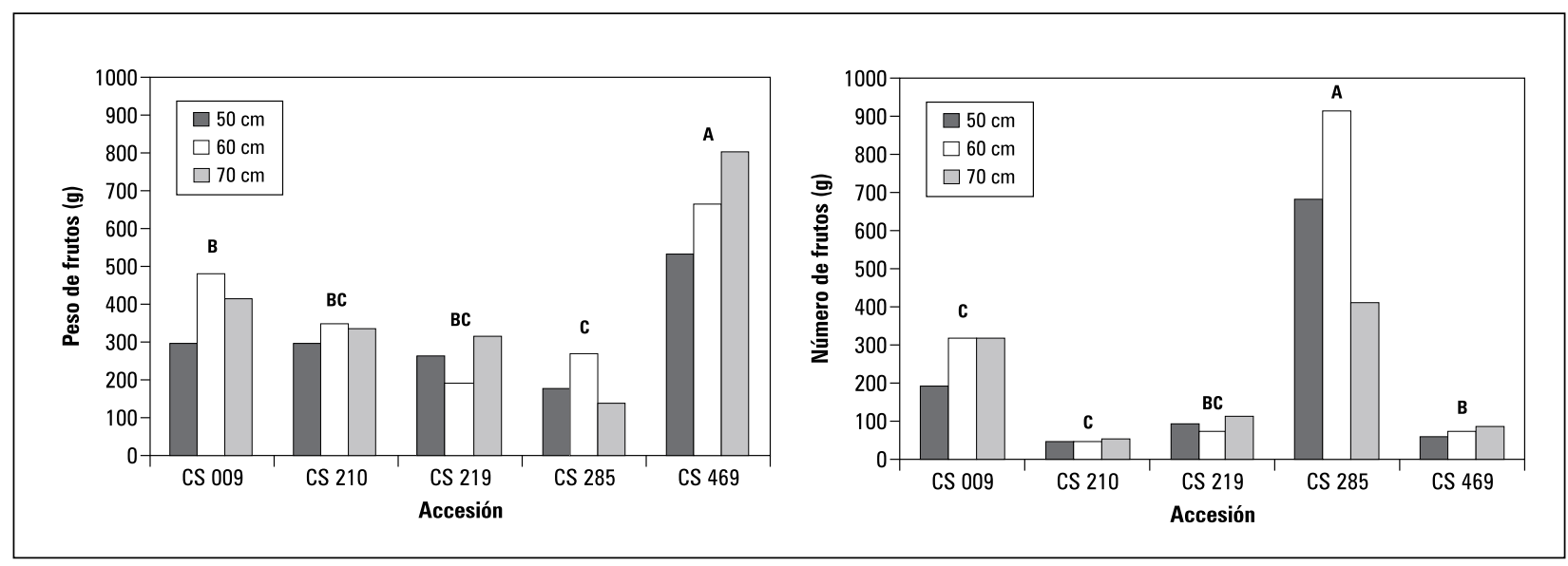

Figura 5. Peso y número de frutos cosechados de cinco accesiones de ají Capsicum spp. con tres distancias de siembra. Las accesiones con letras distintas sobre las columnas indican diferencia significativa según la prueba de $L S D(P \leq 0,05)$.

Entre distancias de siembra no se registraron diferencias significativas en las accesiones ni en la interacción accesión por distancia $(P>0,05)$. Según Perez et al. (2003), en condiciones de alta competencia, las plantas responden fenotípicamente, sintetizando mayor proporción de asimilados para contrarrestar el efecto de competencia por RFA. De acuerdo con Arjona et al. (1992) y Azcón-Bieto y Talón (1993), las diferencias en el peso de los frutos puede atribuirse a la composición genética de los diferentes materiales evaluados porque la variedad tiene una gran influencia sobre la velocidad de crecimiento, el tamaño final y la forma del fruto, además del importante efecto ambiental.

La accesión con mayor contenido de pungencia es la accesión CS 210 (C. chinense), seguida de la CS 219 (C. annuum) y la CS 285 (C. frutescens). En la tabla 2 se muestra que la pungencia en USH de estas accesiones estaba en su orden de mayor a menor (CS 210-CS 469-CS 219- CS285-CS 009). Los resultados de la figura 6 muestran que indistintamente de la densidad de siembra la accesión CS 210 mantuvo el mayor nivel de pungencia, pero la CS 469 que contenía el segundo mayor nivel se ubicó en el cuarto lugar.

Fujikawi et al. (1980) y Calva et al. (2000) anotan que los capsaicionoides son producidos en las glándulas localizadas cerca de la placenta en las paredes del fruto, producto de la ruta del áci- do cinámico. Ésta empieza a acumularse a partir de los $8 \mathrm{~d}$ a $10 \mathrm{~d}$ después de la antesis, aumentando a medida que transcurre la maduración de los frutos para llegar a un máximo cuando éstos se tornan completamente maduros (rojos, naranjas o amarillos) y cayendo drásticamente en la senescencia (Iwai et al., 1979). Barrera et al. (2008) mencionan que al inicio de la senescencia hay una importante pérdida de capsaicionides de los frutos producto de la actividad de las peroxidasas. También mencionan que para accesiones pungentes de ají amazónico los niveles óptimos de síntesis de capsaicina y dihidrocapsaicina se dan durante la etapa que corresponde a la máxima coloración típica de cada una de esas accesiones y coincide con el momento de la recolección. Es probable que la CS 469 haya sido colectada en una etapa temprana de desarrollo porque el cambio de color verde a morado y rojo se da en etapas tempranas de desarrollo.

\section{Radiación solar interceptada}

Las accesiones CS 009, CS 219 y CS 285 mostraron un mayor porcentaje de interceptación (figura 7) en la distancia de siembra de $70 \mathrm{~cm}$, seguida de la de $60 \mathrm{~cm}$ y finalmente la de $50 \mathrm{~cm}$. Midmore et al. (1988) encontraron que una gran cobertura del follaje con la consecuente interceptación de radiación fotosintéticamente activa fue característica en las altas densidades. No se presentaron diferencias entre las condiciones de 


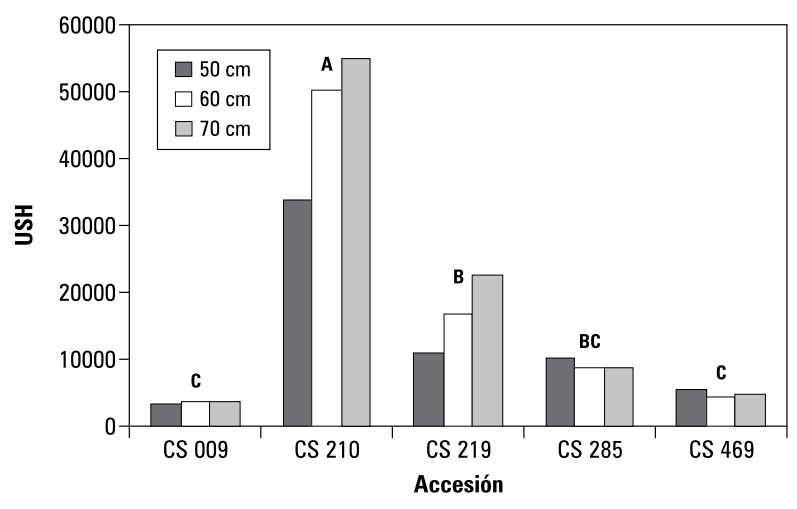

Figura 6. Contenido de capsaicinoides en unidades USH de cinco accesiones de ají Capsicum spp. con tres distancias de siembra. Las accesiones con letras distintas sobre las columnas indican diferencia significativa según la prueba de LSD $(P \leq 0,05)$.

cielo despejado y nublado, al igual que lo encontrado por Ortega-Farias et al. (2004) en tomate.

La radiación interceptada por el dosel mostró diferencias significativas entre distancias de siembra y entre accesiones. La accesión CS 210 presentó los valores significativos más bajos $(P>0,05)$ de porcentaje de radiación absorbida, producto del escaso desarrollo foliar de la planta, y con un tipo de ramificación antocladial con entrenudos largos, muy común en hábito de crecimiento voluble (Nuez y Costa, 1996). Esta disposición reduce la capacidad de interceptar RFA en un nivel superior al 40\%, que fue el común para las demás accesiones.
El mayor porcentaje de interceptación (tabla 6) lo obtuvo la accesión CS 285 (C. frutescens), el cual fue significativo $(P \leq 0,05)$ en los dos estados fenológicos evaluados. Sin embargo, esta accesión presentó los valores más altos de número de frutos y el menor peso de frutos. Ésta accesión se caracteriza por agrupar la mayor capacidad de producción en el estrato superior debido al tamaño reducido de sus frutos, lo cual permite inferir que es un material de alta eficiencia en el uso de la radiación solar, concentrando la mayor cantidad de frutos en este estrato, en el que será mejor la relación fuente-demanda.

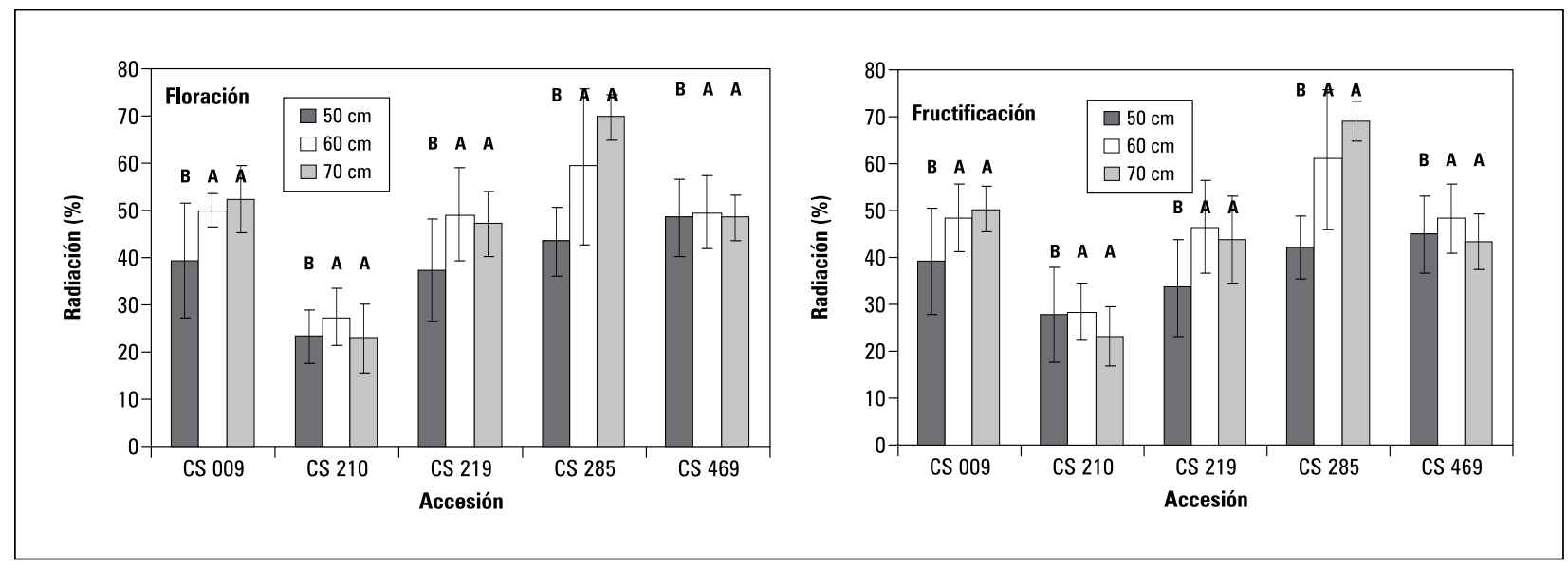

Figura 7. Peso y número de frutos cosechados de cinco accesiones de ají Capsicum spp. con tres distancias de siembra. Las accesiones con letras distintas sobre las columnas indican diferencia significativa según la prueba de LSD $(P \leq 0,05)$. Las barras sobre las columnas indican desviación estándar. 


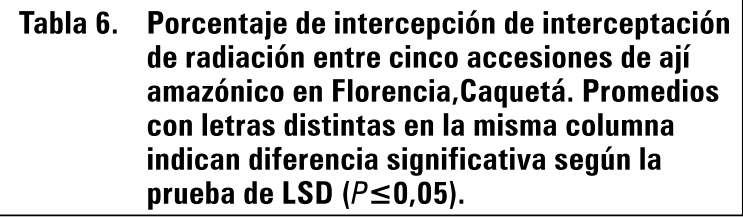

\begin{tabular}{|c|c|c|c|c|}
\hline \multirow{2}{*}{ Accesión } & \multicolumn{2}{|c|}{ Floración } & \multicolumn{2}{|c|}{ Fructificación } \\
\cline { 2 - 5 } & Promedio & & Promedio & \\
\hline CS 009 & 47,270 & b & 45,976 & b \\
\hline CS 210 & 24,497 & c & 26,409 & c \\
\hline CS 219 & 44,515 & b & 41,225 & b \\
\hline CS 285 & 57,522 & a & 57,352 & a \\
\hline CS 469 & 48,805 & b & 45,549 & b \\
\hline
\end{tabular}

\section{CONCLUSIONES}

Los ajíes amazónicos en sus etapas vegetativas presentaron un comportamiento típico de las especies anuales en las que la planta produce biomasa de manera eficiente y sostenida en res- puesta a la capacidad fotosintética. Este periodo va hasta los $33 \mathrm{ddt}$ en promedio, momento a partir del cual se da la curva de crecimiento exponencial, aunque en algunas accesiones se dio de manera más tardía. La interacción entre el genotipo y el ambiente determinó la respuesta poco común obtenida en cuatro de las cinco accesiones porque la arquitectura de la planta en las especies chinense, frutescens y annuum es diferente. La producción y calidad de los frutos no se vio afectada por las densidades de siembra, lo cual es una respuesta de aclimatación de las especies del género Capsicum a las condiciones ambientales de disipación de radiación directa. Larcher (2003) menciona que la luz directa pasa a hojas bajeras, por lo que éstas absorben gran cantidad de luz difusa, y hace suponer adaptación a bajos puntos de compensación de luz, por lo que habría una ventaja en condiciones de alta densidad (Cabezas y Corchuelo, 2005).

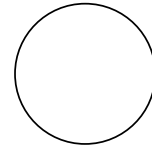

Arjona, H.; M. Montalvo y M. Soto. 1992. Evaluación del comportamiento agronómico de tres híbridos y dos cultivares de pepino cohombro (Cucumis sativus L) bajo condiciones de invernadero en la sabana de Bogotá. Revista Comalfi 19, 11-14.

Azcón-Bieto, J. y M. Talón. 1993. Fisiología y bioquímica vegetal. McGraw-Hill Interamericana de España, Madrid.

Barrera, K.; M.S. Hernández; L.M. Melgarejo; O. Martínez y J.P. Fernández-Trujillo. 2008. Physiological behavior and quality traits during fruit growth and ripening of four Amazonic hot pepper accessions. J. Sci. Food Agric. 88, 847-857.

Cabezas, M. y G. Corchuelo. 2005. Estimación de la interceptación de la radiación solar en papa criolla (Solanum phureja Juz. et Buk.) en tres localidades colombianas. Agron. Colomb. 23(1), 62-73.

Calva, G.; A. Ochoa.; L.E. Rios.; J. Sánchez y V. Martínez. 2000. Catabolismo de capsaicina En: Cultivos de células de Capsicum. Cinvestav. San Pedro Zacatenco, México D.F.

\section{REFERENCIAS BIBLIOGRÁFICAS}

Carrillo, J.C.; F. Jiménez; J. Ruiz; G. Díaz; P. Sánchez; C. Perales y A. Arellanes. 2003. Evaluación de densidades de siembra en Tomate (Lycopersicon esculentum Mill.) en invernadero. Agron. Mesoam. 14(1), 85-88.

Castillo, R.E.; P.J. Arcila.; R.A. Jaramillo y J. Sanabria. 1997. Interceptación de la radiación fotosintéticamente activa y su relación con el área foliar de Coffea arabica. Cenicafe 48(3), 182-194.

Decoteau, D.R. y H.A. Hatt Graham. 1994. Plant spatial arrangement affects growth, yield, and pod distribution of cayenne peppers. HortScience 29(3), 149-151.

Díaz, L.; A. Vitoria y L. Arteaga. 1999. Crecimiento vegetativo del Pimentón en función de la densidad de plantas y edad del cultivo. Bioagro 11(2), 69-73.

Fujikawi, H.; T. Suzuki; S. Osaka y K. Iwai. 1980. Enzymatic formation of capsaicionoid from vanillylamine and iso-type fatty acids by cells free extracts of Capsicum annuum var Karayatsubusa. Agric. Biol. Chem. 44, 2907-2912. 
Gardner, F.P.; R.B. Pearce y R.L. Mitchell. 1985. Physiology of crop plants. Iowa State University, Ames, IA.

Hunt, R. 2003. Plant growth analysis: individual plants. pp. 579-588. En: Thomas, B.; D.J. Murphy y D. Murray (eds.). Encyclopedia of Applied Plant Sciences. Academic Press, London.

IBPGR. 1983. Genetic resources of Capsicum. A global plan action. International Board of Plant Genetic Resources AGPG/ IBPGR/82/12. Roma.

Idinoba, M.E.; P.A. Idinoba y A.S. Gbadegesin. 2002. Radiation interception and its efficiency for dry matter production in the three crop species in the transitional humid zone of Nigeria. Agronomie $22(7), 273-281$.

Iwai, K.; T. Suzuki y H. Fujikawe. 1979. Formation and accumulation or pungent principle of Hot Pepper fruits. Capsaicin and its analogues, in Capsicum annuum var. annuum cv. Karayatsubusa at different growth stages after flowering. Agric. Biol. Chem. 6, 2493-2498

Jaramillo-Robledo, A. 2005. La redistribución de la radiación solar y la lluvia dentro de plantaciones de café (Coffea arabica L.). Rev. Acad. Colom. Cienc. 29(112), 371-382.

Jarma, A.; C. Buitrago y S. Gutiérrez. 1999. Respuesta del crecimiento de la habichuela (Phaseolus vulgaris L. var. Blue Lake) a tres niveles de radiación incidente. Revista Comalfi 26(1-3), 62-73.

Jarma, A.; T. Rengifo y H. Aramendia. 2006. Fisiología de estevia (Stevia rebaudiana) en función de la radiación en el Caribe colombiano. II. Análisis de crecimiento. Agron. Colomb. 24(1), 38-47

Larcher, W. 2003. Physiological plant ecology. $4^{\text {th }}$ ed. Springer-Verlag, Berlin.

Lee, D.W.; S.F. Oberbauer y P. Johnson. 2000. Effects of irradiance and spectral quality on leaf structure and function in seedlings of two southeast asian Hopea (Dipterocarpaceae) species. Amer. J. Bot. 87(4), 447-455.

Mansab A.; D. Jeffers y P. Henderlong. 2003. Interrelationship between leaf area, light interception and growth rate in a soybean-wheat system. Asian J. Plant Sci. 2181, 605-612.

Melgarejo, L.M; F. Rodríguez; M. Giraldo; G. Cardona; M. Celis; J.C. Arias; M. García; L. Quintero; M. Cudris; S. Toquita; I. Monroy; M.E. Rodríguez;
M.C. Duque y J. Tohme. 2004. Caracterización de accesiones del banco de germoplasma de la región amazónica colombiana. pp. 13-26. En: Melgarejo, L.M.; M.S. Hernández; J.A. Barrera y X. Bardales, (eds.). Caracterización y usos potenciales del banco de germoplasma de ají amazónico. Instituto Amazónico de Investigaciones Científicas, Sinchi, Universidad Nacional de Colombia, Gráficas Ducal Ltda., Bogotá.

Méndez, M.A.; G.A. Ligarreto; M.S. Hernández; L.M. Melgarejo y V. González. 2003. Evaluación agronómica de 4 accesiones de ají amazónico del banco de germoplasma ex situ del Instituto Sinchi. Agron. Colomb. 21(3), 165-174.

Méndez, M.A.; G.A. Ligarreto; M.S. Hernández y L.M. Melgarejo. 2004. Evaluación del crecimiento y determinación de índices de cosecha en frutos de cuatro materiales de Ají (Capsicum sp.) cultivados en la Amazonía Colombiana. Agron. Colomb. 22(1), 7-17.

Nuez, F. y J. Costa. 1996. El cultivo de pimientos, chiles y ajíes. Ediciones Mundi-Prensa, Madrid.

Ortega-Farias, S.; R. Calderón; N. Martelli y R. Antonioletti. 2004. Evaluación de un modelo para estimar la radiación neta sobre un cultivo de tomate industrial. Agric. Técnic. 64, 41-49.

Páez, A.; V. Paz y J. López. 2000. Crecimiento y respuestas fisiológicas de plantas de tomate cv. 'Río Grande'. Rev. Fac. Agron. (LUZ) 17(2), 173-184.

Perez, L.; G. Corchuelo y E. Ñustez. 2003. Influencia del espaciamiento entre plantas sobre la morfología y el crecimiento de la papa (Solanum tuberosum L. cv. Parda pastusa) bajo dos ambientes contrastantes. Agron. Colomb. 21(3), 210-219.

Rodríguez, P.L.; R.G. Corchuelo y C.E. Ñústez. 2004. Influencia del espaciamiento entre plantas sobre la morfología y el crecimiento de la papa (Solanum tuberosum L. cv. Parda pastusa) bajo dos ambientes contrastantes. Agron. Colomb. 21(3), 210-219.

Sinan, E.; O. Yemis; C. Kadakal y N. Artik. 2005. Determination of capsaicinoid profile of different chilli peppers grown in Turkey. J. Sci. Food Agr. 85,1435-1438.

Villachica, H. 1996. Frutales y hortalizas promisorias de la Amazonia. Tratado de Cooperación Amazónica, Lima.

Viloria, A.; L. Arteaga y H.A. Rodríguez. 1998. Efecto de la distancia de siembra en las estructuras de la planta del pimentón. Agron. Tropic. 48(4), 413-423. 